\title{
Editorial: Ecology and Evolution of Non-Consumptive Effects in Host-Parasite Interactions
}

\author{
Janet Koprivnikar ${ }^{1 *}$, Sara B. Weinstein ${ }^{2}$, Lien T. Luong ${ }^{3}$ and Julia C. Buck ${ }^{4}$ \\ ${ }^{1}$ Department of Chemistry and Biology, Ryerson University, Toronto, ON, Canada, ${ }^{2}$ School of Biological Sciences, University \\ of Utah, Salt Lake City, UT, United States, ${ }^{3}$ Department of Biological Sciences, University of Alberta, Edmonton, AB, Canada, \\ ${ }^{4}$ Department of Biology and Marine Biology, University of North Carolina Wilmington, Wilmington, NC, United States
}

Keywords: avoidance, natural enemy, parasite, non-consumptive effect, risk, infection

\section{Editorial on the Research Topic}

Ecology and Evolution of Non-Consumptive Effects in Host-Parasite Interactions

\section{INTRODUCTION}

OPEN ACCESS

Edited and reviewed by: Jordi Figuerola, Estación Biológica de Doñana (EBD),

Spain

*Correspondence: Janet Koprivnikar

jkoprivn@ryerson.ca

Specialty section:

This article was submitted to Behavioral and Evolutionary Ecology, a section of the journal

Frontiers in Ecology and Evolution

Received: 17 September 2021 Accepted: 11 October 2021 Published: 29 October 2021

Citation:

Koprivnikar J, Weinstein SB, Luong LT and Buck JC (2021) Editorial: Ecology and Evolution of Non-Consumptive

Effects in Host-Parasite Interactions.

Front. Ecol. Evol. 9:779102.

doi: 10.3389/fevo.2021.779102
The COVID-19 pandemic has vividly illustrated that when a highly contagious, deadly pathogen begins circulating, humans adjust their behavior to reduce their risk of contracting the disease. Such avoidance-induced changes in social interactions, movement, and food acquisition, i.e., "nonconsumptive effects" (NCEs), can be extremely costly to hosts, reducing our GDP, impacting our mental and physical health, and shrinking our birth rates (e.g., Buck and Weinstein, 2020). Furthermore, infection avoidance can trigger cascading effects on other species and the environment. For instance, lockdowns reduced greenhouse gas emissions and caused behavioral changes in various animal species (Bates et al., 2021; Montgomery et al., 2021). But humans are not the only species that experiences infection outbreaks. Animals, too, host a variety of infectious agents, and behaviorally-mediated parasite avoidance is increasingly recognized as widespread and important (e.g., Behringer et al., 2018; Hart and Hart, 2018).

This special issue draws attention to the mounting evidence that parasites and pathogens can impose NCEs on hosts, and the diversity of study systems spanned by the contributed articles speak to the generality of this phenomenon. These provide examples of behavioral mitigation of infection risk by various animals, from mollusks to mammals, as well as considering the consequences of behavioral trait changes, and the evolution of strategies to avoid infection. The inclusion of different host-parasite systems adds considerable phylogenetic breadth to our current state of knowledge-a critical element as we work toward identifying and quantifying the effects exerted by natural enemies irrespective of how they consume their victim (infection in this case). Both invertebrate and vertebrate hosts are investigated, with parasites ranging from helminths and fungi to biting flies, as well as aquatic and terrestrial habitats. The findings of the contributors clearly demonstrate that different animal species engage in behavioral avoidance to reduce their infection risk, and that this can take different forms. In addition, this Research Topic takes a comprehensive view in 


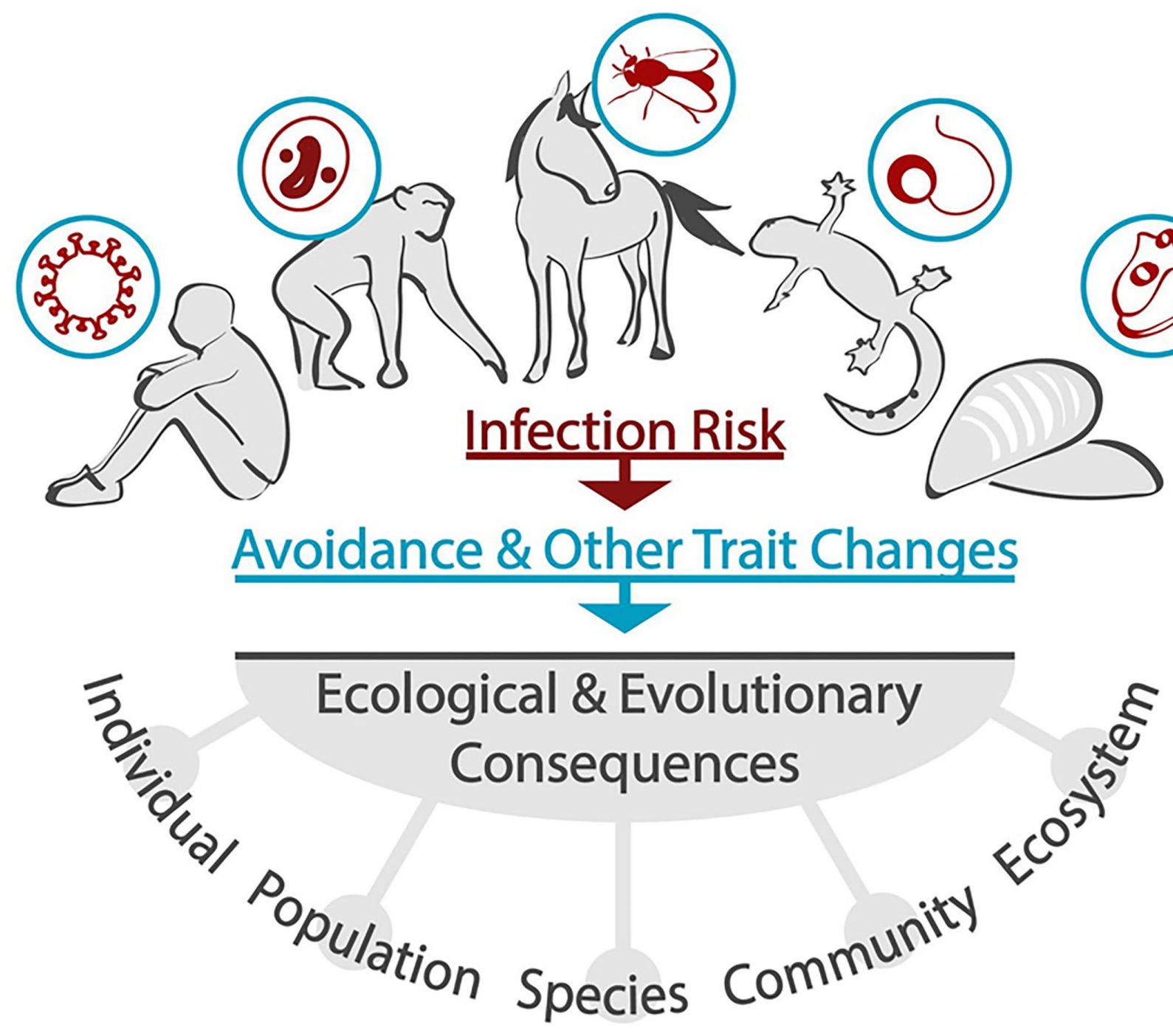

FIGURE 1 | Across diverse hosts and parasites, infection risk generates avoidance via changes in behavior and other traits. Papers in this special topic provide insight into the mechanisms of detection, the range of avoidance behaviors, trade-offs associated with reducing infection risk, and the ecological and evolutionary consequences of avoiding infection. As demonstrated by Selbach and Mouritsen, consequences can occur at a range of scales, from individual (e.g., reduced feeding) to ecosystem (e.g., altered nutrient cycling). Effects at the individual and population levels can also exert selective pressure, acting as feedback on avoidance behaviors to increase or maintain these for any given host-parasite system.

considering the occurrence and consequences of parasite/pathogen-induced NCEs from beginning to end. This starts with detection of risk by potential victims, followed by avoidance behaviors, and some of these studies are the first to show that parasite-induced NCEs can affect host populations, community interactions, and ecosystem structures (Figure 1). Such an integrated viewpoint is necessary to better understand when and why host trait alterations occur in response to the threat posed by parasites and pathogens. As the NCEs of parasites have been largely ignored until relatively recently, we may be severely underestimating the total cost of living in an infectious world. Below we briefly summarize the key messages of the contributed articles, followed by suggestions for future studies that build upon these.

\section{BEHAVIORAL AVOIDANCE OF INFECTION}

Lopes broadly observes that social-distancing measures employed during the COVID-19 pandemic correspond to strategies used by non-human animals, and that we are not alone in using such tactics to reduce infection risk. To this end, Friesen and Detwiler explore the means by which potential hosts detect and avoid transmission risk, particularly in aquatic environments, where potential hosts may use chemical cues (oxylipins) to detect parasitized conspecifics and avoid becoming infected. Working at the interface between aquatic and terrestrial environments, Daversa et al. demonstrate that alpine newts use non-visual cues from parasite-exposed conspecifics to inform habitat avoidance that could reduce their risk of 
Batrachochytrium dendrobatidis infection. However, these newts might experience NCEs as a result of altered habitat use. Sarabian et al. find that bonobos avoid soil- and fecally-contaminated food, with individuals exhibiting the strongest avoidance behaviors the least likely to be infected by Balantioides coli, a parasitic protozoan with an oral-fecal route of transmission. Rubenstein and Feinstein show that horses adjust their daily habitat use to avoid being bitten by blood-drinking flies, basing foraging decisions on the speed of winds that mitigate this risk. As different habitats provide different nutrients, here, parasite avoidance might not require the trade-offs often seen in other systems.

\section{CONSEQUENCES OF PARASITE/PATHOGEN AVOIDANCE}

Selbach and Mouritsen consider how reductions in mussel filtering activity to avoid trematode infection might have far-reaching consequences for aquatic communities, with parasites acting as cryptic ecosystem engineers via NCEs. Koprivnikar et al. examine similarities and differences between host-parasite and plant-invertebrate herbivore systems in terms of natural enemy detection and potential for risk-induced trait alterations. Such comparisons may prove useful for predicting the occurrence and costs of NCEs.

\section{EVOLUTION OF INFECTION AVOIDANCE STRATEGIES}

Amoroso evaluates whether physiological resistance to infection is a useful framework for considering the evolution of behavioral resistance, concluding that there are some benefits, with avoidance behaviors more likely to represent an innate rather than a learned strategy. Poulin et al. develop a "ghost of parasitism past" hypothesis, suggesting that animal species with lower than expected parasite diversity are a good starting point in the search for traces of past parasite-mediated selection. This could help explain the dynamic and inconsistent relationship between the expression of avoidance traits and relative infection risk in comparative analyses across host species. Relatedly, Doherty and Ruehle consider the evolution of avoidance behaviors in an integrated "landscape of peril" containing predators,

\section{REFERENCES}

Bates, A. E., Primack, R. B., Duarte, C. M., and PAN-Environment Working Group (2021). Global COVID-19 lockdown highlights humans as both threats and custodians of the environment. Biol. Conserv. 20:109175. doi: 10.1016/j.biocon.2021.109175

Behringer, D. C., Karvonen, A., and Bojko, J. (2018). Parasite avoidance behaviors in aquatic environments. Philos. Trans. R. Soc. B Biol. Sci. 373:20170202. doi: $10.1098 /$ rstb.2017.0202

Buck, J. C. (2019). Indirect effects explain the role of parasites in ecosystems. Trends Parasitol. 35, 835-847. doi: 10.1016/j.pt.2019.07.007

Buck, J. C., and Weinstein, S. B. (2020). The ecological consequences of a pandemic. Biol. Lett. 6:20200641. doi: 10.1098/rsbl.2020.0641 parasites, and other natural enemies that may exert distinct selective pressures.

\section{CONCLUSIONS}

Articles contributed to this Research Topic have advanced our knowledge of the ecology and evolution of NCEs, but clearly some gaps persist. Specifically, examples of risk-induced trait alterations from a broader range of host and parasite species are needed. The emphasis to date has been on behavioral alterations, but hosts could also alter other traits (e.g., morphology, physiology, development) to avoid parasites. Because the fitness costs of trait alterations are often assumed rather than directly measured (e.g., Luong et al., 2017), we still have little understanding of how consumptive and nonconsumptive effects of parasites compare in terms of frequency and cost. Although a few trait-mediated effects triggered by parasite NCEs have been reported (including in this Research Topic), these pale in comparison to density- and trait-mediated indirect effects stemming from consumption by parasites (Buck, 2019). Furthermore, consequences for ecosystem structure and function remain largely unknown, although it has been shown that predators can elicit such effects. Given that NCEs are ubiquitous yet often overlooked, the true cost of parasitism may be underestimated in many host populations, making this an important area of continued study.

\section{AUTHOR CONTRIBUTIONS}

JK, SW, LL, and JB wrote and edited the article. All authors contributed to the article and approved the submitted version.

\section{FUNDING}

This work was supported by a grant from the Natural Sciences and Engineering Research Council of Canada to JK (04622), and the Ruth L. Kirschstein National Research Service Award to SW (NIH T32AI055434).

\section{ACKNOWLEDGMENTS}

We thank the editorial board at Frontiers in Ecology and Evolution for their support, as well as the many peer reviewers of the contributed articles.

Hart, B. L., and Hart, L. A. (2018). How mammals stay healthy in nature: the evolution of behaviors to avoid parasites and pathogens. Philos. Trans. R. Soc. B Biol. Sci. 373:20170205. doi: 10.1098/rstb.2017.0205

Luong, L. T., Horn, C. J., and Brophy, T. (2017). Mitey costly: energetic costs of parasite avoidance and infection. Physiol. Biochem. Zool. 90, 471-477. doi: $10.1086 / 691704$

Montgomery, R. A., Raupp, J., and Parkhurst, M. (2021). Animal behavioral responses to the COVID-19 quietus. Trends Ecol. Evol. 36, 184-186. doi: $10.1016 /$ j.tree.2020.12.008

Conflict of Interest: The authors declare that the research was conducted in the absence of any commercial or financial relationships that could be construed as a potential conflict of interest. 
Publisher's Note: All claims expressed in this article are solely those of the author and do not necessarily represent those of their affiliated organizations, or those of the publisher, the editors and the reviewers. Any product that may be evaluated in this article, or claim that may be made by its manufacturer, is not guaranteed or endorsed by the publisher.
Copyright $\odot 2021$ Koprivnikar, Weinstein, Luong and Buck. This is an open-access article distributed under the terms of the Creative Commons Attribution License (CC $B Y)$. The use, distribution or reproduction in other forums is permitted, provided the original author(s) and the copyright owner(s) are credited and that the original publication in this journal is cited, in accordance with accepted academic practice. No use, distribution or reproduction is permitted which does not comply with these terms. 